\section{Repeated courses of escalating doses of Nivolumab in refracto- ry Hodgkin lymphoma with recurrent relapses post allografting: $A$ safe and effective treatment approach}

Panayotis Kaloyannidis, ${ }^{1}$

Eshrak Al Shaibani, ${ }^{1}$ Asif Moinnudin, ${ }^{2}$ Khalid Al Anezi, ${ }^{1}$ Hani Al Hashmi ${ }^{1}$

Departments of ${ }^{1}$ Adult Hematology and Stem Cell Transplantation, ${ }^{2}$ Medical Imaging, King Fahad Specialist

Hospital, Dammam, Saudi Arabia

\begin{abstract}
For patients with Hodgkin Lymphoma (HL) who experience relapse post allogeneic stem cell transplantation, limited treatment options exist, and the ultimate outcome is poor. Recently, the programmed cell death protein-1 (PD-1) inhibitors have shown remarkable efficacy in patients with refractory/relapsed HL, also demonstrating an acceptable safety profile. However, due to effects on T-cell activity, the use of PD-1 inhibitors post allografting may potentially increase the risk of treatment-emergent graft versus host disease. We herein report the clinical course of a patient who experienced multiple relapses of HL post allogeneic stem cell transplantation. He failed several treatment modalities but he responded to escalating doses of the PD-1 inhibitor nivolumab, given at two different treatment time points, also demonstrating minimal and easily manageable toxicity.
\end{abstract}

\section{Introduction}

In patients allografted for refractory Hodgkin Lymphoma (HL) who experience disease recurrence post allogeneic hematopoietic stem cell transplantation (alloSCT), the traditional immunomodulatory approaches [reduction or discontinuation of immunosuppressive treatment (IST) with or without donor's lymphocytes infusions (DLIs)] are only partially effective and are usually accompanied with high incidence of induced graft-versus-host disease (GvHD), while chemotherapeutic approaches are mostly unsuccessful. ${ }^{1,2}$ It has been shown that HL cells have a unique biologic dependence on the programmed cell death protein-1 (PD-1), which is expressed on activated T-cells, and other lymphoid and myeloid cells. ${ }^{3,4}$ Nivolumab (Opdivo ${ }^{\circledR}$, Bristol-Meyers Squibb), a fully humanized IgG4 anti-PD-1 monoclonal antibody, has been approved by the US Food and Drug Administration and the European Medicines Agency for the treatment of adults with relapsed/refractory classical Hodgkin Lymphoma (cHL) after autoHSCT and Brentuximab Vendotin (BV) treatment. 5,6

For patients who have relapsed HL post allograft, the inhibition of the PD-1 pathway, might theoretically enhance the graft versus HL (GvHL) effect by upregulating the activation of donor-derived T-cells however, the increased alloreactivity of donor Tcells may also promote severe treatmentemergent GvHD (te-GvHD), thus contributing to increased risk for morbidity and mortality. ${ }^{7}$

Herein, we describe the clinical course of a patient with HL, who experienced early relapse post alloSCT and failed to achieve disease control after immunomodulatory approaches (including anti-CD30 infusion), but responded twice to Nivolumab treatment. To reduce the risk of GvHD without negatively affecting the GvHL effect, we treated the patient with escalating doses of Nivolumab.

\section{Case Report}

A young male patient, diagnosed at the age of 11 years with nodular sclerosis cHL and stage IIIBS, was initially treated in a pediatric center with the ABVD/COPP regimen. After 6 cycles of treatment he failed to achieve remission and therefore a Bortezomib-based salvage regimen was given, followed by autoSCT after BEAM conditioning regimen (Table 1). Nine months post autoSCT, he experienced disease progression with liver, marrow and multiple sites of lymph node involvement and he subsequently received BV (Table 1). Although there was an initial partial response, eventually, 8 months later, the disease progressed again, and the combination of BV with Bendamustine (BvB) was given as previously described by La Casce et al. ${ }^{8}$ After 3 cycles of the BvB combination, the disease was assessed to be in very good partial remission (VGPR) and since he had a suitable fully matched sibling donor, he proceeded to alloSCT after a reduced-intensity conditioning regimen, consisting of thiotepa, fludarabine and cyclophosphamide (Table 1). Cyclosporine plus mycophenolate mofetil were administered as GvHD prophylaxis. He engrafted successfully and experienced no GvHD during
Correspondence: Panayotis Kaloyannidis, Adult Hematology \& Stem Cell Transplantation Department, King Fahad Specialist Hospital, Ammar Bin Thabit St, Al Muraikabat, 314444, Dammam, Saudi Arabia. Tel.: +966503301174 - Fax: +966138442222. E-mail: pkaloyannidis@yahoo.gr, kalogiannidi.panagiotis@kfsh.sa

Key words: allogeneic hematopoietic stem cell transplantation, Hodgkin lymphoma, programmed death-1 (PD-1) inhibitors.

Contributions: PK, conception and design of the work; analysis and interpretation of data; writing and revising the manuscript approved the version to be published. ES, contributed to the design of the work and data acquisition. $\mathrm{AM}$, contributed to the data acquisition. KA, HH, AM, ES, PK, critically reviewed the manuscript for important intellectual content and approved the version to be published, accountable for all aspects of the work in ensuring that questions related to the accuracy or integrity of any part of the work are appropriately investigated and resolved.

Conflict of interest: The authors declare no potential conflict of interest.

Funding: None.

Ethics approval: It has been received.

Consent to publication: It has been received.

Availability of data and materials: Available by authors.

Received for publication: 6 July 2020

Revision received: 9 December 2020.

Accepted for publication: 1 February 2021

This work is licensed under a Creative Commons Attribution-NonCommercial 4.0 International License (CC BY-NC 4.0).

(C) Copyright: the Author(s), 2021

Licensee PAGEPress, Italy

Hematology Reports 2021; 13:8780

doi:10.4081/hr.2021.8780

the early post-transplant period. Three months later, the disease progressed (first relapse post alloSCT), and in addition to IST discontinuation, BV at standard doses was given. With this treatment modality, partial disease control was achieved. However, the patient developed induced GvHD. Steroids plus cyclosporine were initiated, but the GvHD proved to be steroiddependent, therefore the patient continued on steroid-based treatment. Four months later (16 months post allografting), while he was on double IST, he complained of cough and fever. High resolution computed tomography (HRCT) along with lung biop- 
sy confirmed second disease progression post alloSCT (Figure 1A). IST was tapered and Nivolumab was chosen as the next treatment option, since the patient fulfilled the criteria for Nivolumab initiation as per FDA approved indications. ${ }^{5,6}$ PD-1 expression was not evaluated on the tissue biopsy because this test was not available in our center at that time. Although the standard dose for Nivolumab in adult patients is 240 mg every two weeks, because our patient was a young adolescent and underweight $(42 \mathrm{~kg})$, we chose to treat him with at the dose of $3 \mathrm{mg} / \mathrm{kg}$ every two weeks in order to minimize treatment-related toxicity and risk of te-GvHD. ${ }^{9}$ After 2 cycles, significant clinical improvement was noticed. However, the patient complained of severe abdominal pain and diarrhea. Colonic biopsies confirmed te-GvHD; no other organ involvement. The IST doses were increased while Nivolumab dose was reduced to 2 $\mathrm{mg} / \mathrm{kg}$ every 3 weeks. After two additional cycles at the aforementioned dose, significant disease improvement according to CT criteria was documented (Figure 1B), and the patient became completely asymptomatic from the respiratory and gastrointestinal systems. At this time point, patient's family asked for a second opinion from another center. Following the new medical advice, nivolumab treatment was held and the patient continued only IST to control the residual GvHD. Six months after Nivolumab discontinuation, he presented to our center with the third relapse post alloSCT (Figure 2A). He was re-treated with Nivolumab, and in order to avoid severe toxicities and to minimize the risk of severe te-GvHD, we used escalating doses of Nivolumab, starting from $1.5 \mathrm{mg} / \mathrm{kg}$ every 2 weeks, with a planned dose increase of $0.5 \mathrm{mg} / \mathrm{kg}$ every 2 cycles, if the previous dose was well tolerated. After 8 cycles he achieved a complete metabolic response (mCR) according to $\mathrm{PET} / \mathrm{CT}$ criteria (Figure 2B). He continued on escalating doses of Nivolumab and since no treatmentrelated toxicity was noticed, he finally was able to receive the maximum dose of 3 $\mathrm{mg} / \mathrm{kg}$. He was also on low dose IST (tacrolimus $1 \mathrm{mg}$ daily and prednisone 10 mg every other day) to minimize the risk of the te-GvHD. After the $15^{\text {th }}$ infusion of the treatment, he developed diarrhea. New colon biopsies revealed non-specific colitis changes, and the symptoms attributed to the well-described adverse reaction of the check-point inhibitors. ${ }^{10}$ Since the patient was in a prolonged remission (7 months
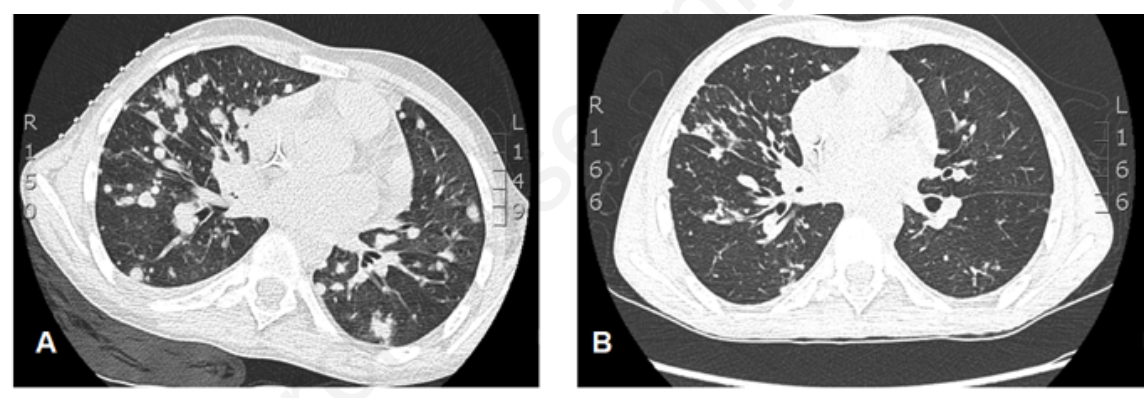

Figure 1. HRCT during the first course of Nivolumab treatment. A) Before Nivolumab administration HRCT showed multiple nodules in the lungs bilaterally. The CT guided biopsy confirmed relapse of cHL. B) After the first 4 cycles of Nivolumab administration the HRCT showed excellent response since the majority of the malignant nodules have been disappeared. HRCT: High resolution computed tomography, cHL: classical Hodgkin Lymphoma.

Table 1. Summary of treatment.

$\begin{array}{llll}\text { Regimen } & \text { Drugs and dosage } & \text { Courses } & \text { Response } \\ \text { ABVD/COPP } & \text { Doxorubicin: } 25 \mathrm{mg} / \mathrm{m}^{2} \text { for } 2 \text { days }(1 \& 15) & 6 & \text { Induction failure } \\ & \text { Bleomycin: } 10 \mathrm{mg} / \mathrm{m}^{2} \text { for } 2 \text { days }(1 \& 15) & \end{array}$

Bleomycin: $10 \mathrm{mg} / \mathrm{m}^{2}$ for 2 days (1\&15)

Vinblastine: $6 \mathrm{mg} / \mathrm{m}^{2}$ for 2 days (1\&15)

Dacarbazine: $400 \mathrm{mg} / \mathrm{m}^{2}$ for 2 days (1\&15)

Cyclophosphamide: $200 \mathrm{mg} / \mathrm{m}^{2}$ for 2 days $(1 \& 8)$

Vincristine: $1.5 \mathrm{mg} / \mathrm{m}^{2}$ for 2 days (1\&8)

Procarbazine: $100 \mathrm{mg} / \mathrm{m}^{2}$ for 10 days

Prednisone: $1 \mathrm{mg} / \mathrm{kg}$ for 10 days

\begin{tabular}{llll} 
AHOD0521 & Ifosfamide: $3000 \mathrm{mg} / \mathrm{m}^{2}$ for 4 days (1-4) & 2 & Very good partial response \\
& Vinorelbine: $25 \mathrm{mg} / \mathrm{m}^{2}$ for 2 days (1\&5) & & \\
& Bortezomib: $1.2 \mathrm{mg} / \mathrm{m}^{2}$ for 3 days $(1,4 \& 8)$ & & \\
\hline BEAM & Carmustin (BCNU): $300 \mathrm{mg} / \mathrm{m}^{2}$ for 1 day (D-6) & & \\
& Etoposide: $100 \mathrm{mg} / \mathrm{m}^{2}$ for 4 days (D-5 to -2$)$ & & \\
& Cytarabine: $200 \mathrm{mg} / \mathrm{m}^{2}$ for 4 days (D- 5 to -2$)$ & & Disease progression: 9 months post autoSCT \\
Bv & Melphalan: $160 \mathrm{mg} / \mathrm{m}^{2}$ for 1 days (D-1) & 1 & Disease progression \\
\hline BvB & Brentuximab vedotin: $1.8 \mathrm{mg} / \mathrm{m}^{2}$ for 1 day & 8 & Very good partial response
\end{tabular}

Thiotepa, fludarabine and cyclophosphamide Thiotepa: $7 \mathrm{mg} / \mathrm{m}^{2}$ for 1 day (D-7),

Fludarabine: $30 \mathrm{mg} / \mathrm{m}^{2}$ for 3 days (D-5 to -3 )

Cyclophosphamide: $50 \mathrm{mg} / \mathrm{m}^{2}$ for 2 days (D-5 \& -4) $\quad 1 \quad$ Disease progression: 3 months post alloSCT 
patients who experience relapse after alloSCT demonstrate an exhausted $\mathrm{T}$ cell phenotype, having also a low capacity to produce cytokines necessary for T-cell cytotoxic activity. ${ }^{11-13}$ The well-documented overexpression of PD-1 molecules on circulating lymphocytes and HL cells at the time of relapse, suggests that alterations in signaling across the PD1-synapse could be at least an additional pathway for the post alloSCT immune evasion. ${ }^{11}$ Based on this knowledge, the PD-1/PDL-1 pathway inhibitors could be an effective alternative treatment for HL patients who experience relapse after alloSCT.

Our patient had disease refractory to multiple treatment approaches including autoSCT, alloSCT and antiCD30 monoclonal antibody administration; he eventually achieved mCR after Nivolumab treatment. Timmerman et al. reported the results of Nivolumab treatment in patients with HL who had previously treated with Brentuximab after autoSCT failure. After a median follow-up of 15.4 months (minimum 12 months), the overall response rate (ORR) was 68\%, the CR incidence was $8 \%$, while the 1-year progression-free and overall survival were $54 \%$ and $94 \%$, respectively. ${ }^{14}$ The experience of Nivolumab for relapsed disease post alloSCT is limited and based on small series of patients or case reports. Two retrospective studies reported promising results with ORR of $80 \%$ and $95 \%$ and high rates of durable complete remissions $42 \%$ and $50 \%{ }^{15-17}$ It is notewor-

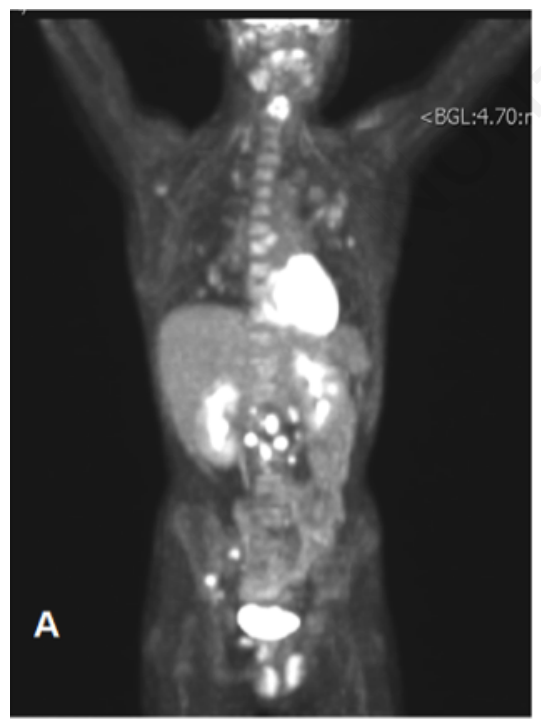

thy that the published ORR for patients who received PD-1 inhibitors for relapsed HL post alloSCT are higher as compared to the response rates that have been observed in patients who were treated with PD-1 inhibitors for disease recurrence post autoSCT or conventional chemotherapy. A plausible explanation for this efficacy inferiority in the autoSCT/conventional chemotherapy setting, could be that the PD1 inhibitor acts on patient T-cells and therefore, either intrinsic patient's lymphocytes deficiency or the previous exposure to chemotherapy, adversely affect the immune response despite priming by PD-1 inhibitor. Oppositely, in the allograft setting, the PD1 inhibitor acts on healthy donor-derived Tcells which are also naïve to chemotherapy.

A major complication of Nivolumab administration after alloSCT, apart from the other common side effects, is the increased donor-derived T-cell alloreactivity, thus resulting in te-GvHD. ${ }^{10,15-18}$ Our patient, after the first 2 cycles of Nivolumab at the dose of $3 \mathrm{mg} / \mathrm{kg}$, developed severe gut teGvHD which was effectively controlled with IST treatment plus Nivolumab dose reduction. It is worth mentioning that prior to Nivolumab treatment, the patient had already experienced induced-GvHD which is a well-known predisposing factor for GvHD flare after PD-1 inhibitor treatment. ${ }^{18}$ So far, there are no definite guidelines regarding the dosing and the duration of treatment with PD-1 inhibitors post allograft. In a prospective phase $\mathrm{I} / \mathrm{Ib}$ multicen-

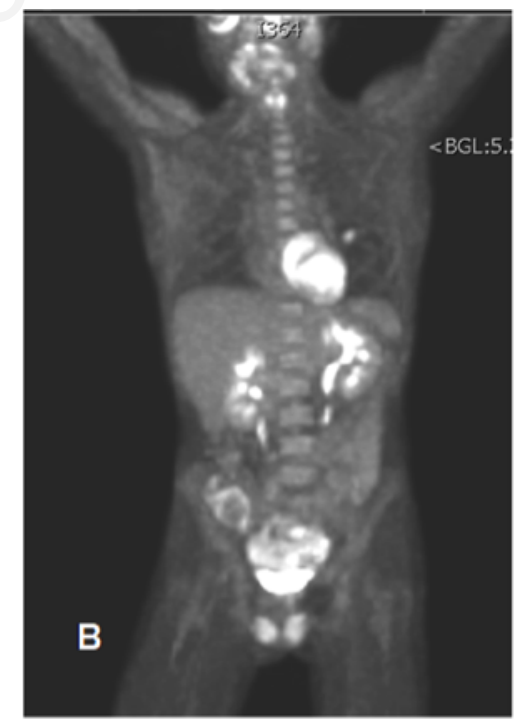

Figure 2. PET/CT scan during the second course of Nivolumab administration. A) Six months after Nivolumab discontinuation the PET/CT showed disease recurrence in the lungs, axillary and retroperitoneal lymph nodes. B) After 8 cycles of Nivolumab readministration, mCR was documented according to PET/CT criteria. PET/CT: Positron emission tomography/computed tomography, mCR: metabolic complete remission. ter study, 8 patients were treated with Nivolumab after allograft for relapsed HL (6 patients at $1 \mathrm{mg} / \mathrm{kg}$ and 2 patients at 0.5 $\mathrm{mg} / \mathrm{kg}$ ). In the $1 \mathrm{mg} / \mathrm{kg}$ cohort there were 2 non-relapse related deaths while 2 patients experienced severe chronic GVHD. On the contrary, no significant toxicities have been observed in the $0.5 \mathrm{mg} / \mathrm{kg}$ cohort of patients. ${ }^{19}$ Onizuca et al. published the clinical course of a patient who received Nivolumab at escalating doses for relapsed HL post alloSCT, starting from $0.5 \mathrm{mg} / \mathrm{kg}$. The dose-escalating ratio was $100 \%$ every 4 cycles, with the maximum reached dose of $2 \mathrm{mg} / \mathrm{kg}$; finally, due to te-GvHD occurrence, the dose was reduced to $1 \mathrm{mg} / \mathrm{kg}$. In terms of the primary disease response, the authors reported that only VGPR was achieved. ${ }^{20}$ In a recent report, Herbaux et al. recommend initiating Nivolumab at a low dose $(0.5 \mathrm{mg} / \mathrm{kg})$ followed by escalating doses if no te-GvHD occurs. ${ }^{21}$ In our case, during the second course of nivolumab treatment, we chose to start with a higher dose $(1,5 \mathrm{mg} / \mathrm{kg})$, followed by a lower escalating ratio $(0.5 \mathrm{mg} / \mathrm{kg}$ every $2-3$ cycles $)$ if the medication was well tolerated. By using this treatment schedule, the patient was able to receive in total 15 cycles and after the $7^{\text {th }}$ cycle/infusion he reached the maximum Nivolumab dose of $3 \mathrm{mg} / \mathrm{kg}$ without any major complication. Interestingly, the efficacy of Nivolumab was robust even at the lower doses, as our patient, during the second escalating Nivolumab course, achieved mCR after the $7^{\text {th }}$ infusion (ranges of the given doses $1.5-2 \mathrm{mg} / \mathrm{kg}$ ).

It has not been reported in the literature whether previous exposure to Nivolumab adversely affects its efficacy or exacerbates toxicity in the case of subsequent administration. Our patient received two nivolumab courses with an interval period of 6 months, and both times he responded successfully, suggesting that at least in certain cases, Nivolumab re-administration retains its efficacy even if malignant cells have been previously exposed to it.

\section{Conclusions}

In this heavily pretreated patient with refractory disease, Nivolumab, given at intermediate dose followed by low rate of dose escalation, was a well-tolerated treatment, offering also the desired efficacy leading to $\mathrm{mCR}$ attainment. Documentation of $\mathrm{mCR}$ even after previous exposure to Nivolumab, merits further investigation. Because of the limited existing data, the treatment duration and the exact dose of PD-1 inhibitors in the post alloSCT setting 
have not been clarified yet and therefore, well-designed prospective studies with large series of patients are warranted.

\section{References}

1. Tsirigotis P, Danylesko I, Gkirkas K, et al. Brentuximab vedotin in combination with or without donor lymphocyte infusion for patients with Hodgkin lymphoma after allogeneic stem cell transplantation. Bone Marrow Transplant 2016;51:1313-7.

2. Sala E, Crocchiolo R, Gandolfi S, et al. Bendamustine combined with donor lymphocytes infusion in Hodgkin's lymphoma relapsing after allogeneic hematopoietic stem cell transplantation. Biol Blood Marrow Transplant 2014;20:1444-7.

3. Ansell SM, Lesokhin AM, Borrello I, et al. PD-1 blockade with Nivolumab in relapsed or refractory Hodgkin's lymphoma. N Engl J Med 2015;372:311-9.

4. Armand P, Shipp MA, Ribrag V, et al. Programmed death-1 blockade with pembrolizumab in patients with classical Hodgkin lymphoma after brentuximab vedotin failure. J Clin Oncol 2016;34:3733-9.

5. Kasamon YL, de Claro RA, Wang Y, et al. FDA Approval Summary: Nivolumab for the Treatment of Relapsed or Progressive Classical Hodgkin Lymphoma. Oncologist 2017; 22:585-91.

6. Armand P, Engert A, Younes A, et al. Nivolumab for Relapsed/Refractory Classic Hodgkin Lymphoma After Failure of Autologous Hematopoietic Cell Transplantation: Extended FollowUp of the Multicohort Single-Arm Phase II CheckMate 205 Trial. J Clin Oncol 2018;36:1428-39.
7. Armand P. Immune check-point blockade in hematologic malignancies. Blood 2015;125:3393-400.

8. LaCasce AS, Bociek RG, Sawas A, et al. Brentuximab vedotin plus bendamustine: a highly active first salvage regimen for relapsed or refractory Hodgkin lymphoma. Blood 2018;132: 40-8.

9. Davis KL, Fox E, Merchant MS, et al. Nivolumab in children and young adults with relapsed or refractory solid tumours or lymphoma (ADVL1412): a multicentre, open-label, single-arm, phase 1-2 trial. Lancet Oncol 2020;21:541-50

10. Postow MA. Managing Immune Checkpoint-Blocking Antibody Side Effects. Am Soc Clin Oncol Educ Book 2015:76-83.

11. Kong Y, Zhang J, Claxton DF, et al. PD1(hi)TIM-3(+) T cells associate with and predict leukemia relapse in AML patients post allogeneic stem cell transplantation. Blood Cancer J 2015;5:e330.

12. Norde WJ, Maas F, Hobo W, et al. PD1/PD-L1 interactions contribute to functional T-cell impairment in patients who relapse with cancer after allogeneic stem cell transplantation. Cancer Res 2011;71:5111-22.

13. Schnorfeil FM, Lichtenegger FS, Emmerig K, et al. T cells are functionally not impaired in AML: increased PD-1 expression is only seen at time of relapse and correlates with a shift towards the memory $\mathrm{T}$ cell compartment. J Hematol Oncol 2015;8:93.

14. Timmerman JM, Engert A, Younes A. et al. Checkmate 205 update with minimum 12-Month follow up: A Phase 2 Study of Nivolumab in Patients with Relapsed/Refractory Classical Hodgkin Lymphoma. Blood 2016;128:1110.
15. Haverkos BM, Abbott D, Hamadani M, et al. PD-1 blockade for relapsed lymphoma post-allogeneic hematopoietic cell transplant: high response rate but frequent GVHD. Blood 2017;130:2218.

16. Herbaux C, Gauthier J, Brice P, et al. Efficacy and tolerability of Nivolumab after allogeneic transplantation for relapsed Hodgkin lymphoma. Blood 2017;129:2471-8.

17. Godfrey J, Bishop MR, Syed S, et al. PD-1 blockade induces remissions in relapsed classical Hodgkin lymphoma following allogeneic hematopoietic stem cell transplantation. J Immunother Cancer 2017;5:11.

18. Armand P, Engert A, Younes A, et al. Nivolumab for Relapsed/Refractory Classic Hodgkin Lymphoma After Failure of Autologous Hematopoietic Cell Transplantation: Extended FollowUp of the Multicohort Single-Arm Phase II CheckMate 205 Trial. J Clin Oncol 2018;36:1428-39.

19. Davids MS, Kim HT, Costello C, et al. Optimizing check-point blockade as a Treatment for relapsed hematologic malignancies after allogeneic hematopoietic cell transplantation. Blood 2017;130:275.

20. Onizuka M, Kojima M, Matsui K, et al. Successful treatment with low-dose Nivolumab in refractory Hodgkin lymphoma after allogeneic stem cell transplantation. Int J Hematol 2017;106:1415

21. Herbaux C, Merryman R, Devine S, et al. Recommendations for managing PD-1 blockade in the context of allogeneic HCT in Hodgkin lymphoma: taming a necessary evil. Blood 2018;132:9-16. 